\title{
Predicting cancer malignancy and proliferation in glioma patients: intra-subject inter-metabolite correlation analyses using MRI and MRSI contrast scans
}

\author{
Changliang Su ${ }^{1}$, Shihui $\mathrm{Li}^{2}$, Xiaowei Chen ${ }^{3}$, Chengxia Liu ${ }^{2}$, Mehran Shaghaghi ${ }^{4}$ Jingjing Jiang ${ }^{2}$, \\ Shun Zhang ${ }^{2}$, Yuanyuan Qin ${ }^{2}$, Kejia Cai ${ }^{4}$ \\ ${ }^{1}$ Department of Medical Imaging, Sun Yat-sen University Cancer Center, State Key Laboratory of Oncology in South China, Collaborative Innovation \\ Center for Cancer Medicine, Guangzhou, China; ${ }^{2}$ Department of Radiology, Tongji Hospital, Tongji Medical College, Huazhong University of \\ Science and Technology, Wuhan, China; ${ }^{3}$ Department of Radiology, The Central Hospital of Wuhan, Tongji Medical College, Huazhong University \\ of Science and Technology, Wuhan, China; ${ }^{4}$ Department of Radiology, the University of Illinois at Chicago, Chicago, Illinois, USA
}

Correspondence to: Yuanyuan Qin. No. 1095, JieFang Avenue, Wuhan 430030, China. Email: qinyuanyuan-1021@163.com.

Background: The non-invasive characterization of glioma metabolites would greatly assist the management of glioma patients in the clinical setting. This study investigated the applicability of intra-subject inter-metabolite correlation analyses for differentiating glioma malignancy and proliferation.

Methods: A total of 17 negative controls (NCs), 39 low-grade gliomas (LGGs) patients, and 25 highgrade gliomas (HGGs) subjects were included in this retrospective study. Amide proton transfer (APT) and magnetization transfer contrast (MTC) imaging contrasts, as well as total choline/total creatine ( $\mathrm{tCho} / \mathrm{tCr}$ ) and total $\mathrm{N}$-acetylaspartate/total creatine $(\mathrm{tNAA} / \mathrm{tCr})$ ratios quantified from magnetic resonance spectroscopic imaging (MRSI) were co-registered voxel-wise and used to produce three intra-subject inter-metabolite

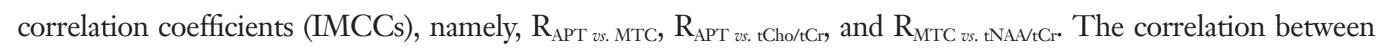
the IMCCs and tumor grade and Ki-67 labeling index (LI) for tumor proliferation were explored. The differences in the IMCCs between the three groups were compared with one-way analysis of variance (ANOVA). Finally, regression analysis was used to build a combined model with multiple IMCCs to improve the diagnostic performance for tumor grades based on receiver operator characteristic curves.

Results: Compared with the NCs, gliomas showed stronger inter-metabolic correlations. $\mathrm{R}_{\mathrm{APT} \text { vs. MTC }}$ was significantly different among the three groups (NC vs. LGGs vs. HGGs: $-0.18 \pm 0.38 v s$. $-0.40 \pm 0.34 v s .-0.70 \pm 0.29$, $\mathrm{P}<0.0001)$. No significant differences were detected in $\mathrm{R}_{\mathrm{MTC} v \text { s. } \mathrm{ANAA} / \mathrm{tCr}}$ among the three groups. $\mathrm{R}_{\mathrm{APT} \text { vs. MTC }}$ and $\mathrm{R}_{\mathrm{APT} \text { vs. tCho/tCr }}$ correlated significantly with tumor grade $(\mathrm{R}=-0.41, \mathrm{P}=0.001$ and $\mathrm{R}=0.448, \mathrm{P}<0.001$, respectively). However, only $\mathrm{R}_{\mathrm{APT} \text { vs. MTC }}$ was mildly correlated with Ki-67 $(\mathrm{R}=-0.33, \mathrm{P}=0.02)$. $\mathrm{R}_{\mathrm{APT} v \text { s. MTC }}$ and $\mathrm{R}_{\mathrm{APT} \text { vs. tCho/tCr }}$ achieved areas under the curve (AUCs) of 0.754 and 0.71 , respectively, for differentiating NCs from gliomas; and 0.77 and 0.78 , respectively, for differentiating LGGs from HGGs. The combined multi-IMCCs model improved the correlation with the $\mathrm{Ki}-67 \mathrm{LI}(\mathrm{R}=0.46, \mathrm{P}=0.0008)$ and the tumor-grade stratification with AUC increased to 0.85 (sensitivity: $80.0 \%$, specificity: $79.5 \%$ ).

Conclusions: This study demonstrated that glioma patients showed stronger inter-metabolite correlations than control subjects, and the IMCCs were significantly correlated with glioma grade and proliferation. The multiIMCCs combined model further improved the performance of clinical diagnosis.

Keywords: Magnetic resonance imaging (MRI); magnetization transfer contrast imaging (MTC imaging); brain tumor; tumor grading; cell proliferation

Submitted Oct 17, 2020. Accepted for publication Mar 18, 2021.

doi: 10.21037/qims-20-1163

View this article at: http://dx.doi.org/10.21037/qims-20-1163 


\section{Introduction}

Glioma is the most common malignant brain tumor. It is associated with a high post-surgical disability rate and a high mortality rate, resulting in an enormous healthcare system burden (1). The current standard treatment regimen involves maximum extent resection plus radiochemotherapy following adjuvant chemotherapy. However, this is not sufficient to significantly prolong a patient's overall survival (2). Obvious substance metabolism changes and accumulation characterize the rapid growth of gliomas compared to normal brain tissue. High-grade gliomas (HGGs) typically have high proliferating rates and possess high and active metabolite transformation rates; thus, they have a distinct metabolic profile compared to lowgrade gliomas (LGGs) (3). Many metabolites play a vital role in maintaining tumor malignancy, and invasiveness, and quantitative metabolic markers are necessary to explore tumor subtypes' characteristics. Understanding the correlations between metabolites and tumor subtypes may help develop personalized therapies targeting unique tumor metabolic profiles (4).

PET-CT (positron emission tomography-computed tomography) is the most common non-invasive tool for imaging in vivo metabolism. With multiple radiolabeled tracers, such as ${ }^{18} \mathrm{~F}$-fluorodeoxyglucose $\left({ }^{18} \mathrm{~F}\right.$-FDG), ${ }^{1} \mathrm{C}$-methionine, ${ }^{18} \mathrm{~F}$-fluoroethyltyrosine $\left({ }^{18} \mathrm{~F}\right.$-FET), and ${ }^{18} \mathrm{~F}$-fluorothymidine $\left({ }^{18} \mathrm{~F}\right.$-FLT), PET provides significant clinical benefits in detecting anaerobic glycolysis and metabolism of amino acids and nucleosides for protein and DNA synthesis in cancer cells (5). Such quantitative information might help forecast variations in genetic, epigenetic, and proteomic factors or variations in invasion, proliferation, and immune-infiltration in gliomas $(5,6)$. However, the need for radiant nuclear contrast agents and their time limitations mean PET-CT is not as convenient as magnetic resonance imaging (MRI) in routine clinical applications. Moreover, the ability of ${ }^{18} \mathrm{~F}$-DOPA $\left({ }^{18} \mathrm{~F}-\mathrm{f}\right.$ luorodihydroxyphenylalanine) uptake parameters from PET-CT to reflect molecular and histological features is limited, and the prediction of prognosis is inefficient (7). In contrast, MRI markers can better reflect glioma biological features and predict a patient's progression-free survival $(8,9)$.

Advanced MRI sequences, such as proton magnetic resonance spectroscopic imaging (MRSI) and chemical exchange saturation transfer (CEST) imaging, provide quantitative parameters reflecting tissue metabolism at the molecular level (10-12). Magnetic resonance (MR) spectroscopy allows non-invasive detection of total choline (tCho), total $\mathrm{N}$-acetylaspartate (tNAA), and total creatine $(\mathrm{tCr})$ levels in cerebral tissue. Amide proton transfer imaging (APT) contrast, a type of CEST, detects cellular mobile proteins and peptides. Semi-solid magnetization transfer contrast (MTC) quantifies the number of macromolecular substances, such as structural proteins and carbohydrates (13-15). Increased metabolites and varied metabolism typically accompany the rapid growth of gliomas. The in vivo detection of these metabolic parameters with MRI/MRSI may allow the non-invasive quantification of key metabolites associated with tumor malignancy, tumor grades, proliferation, or genetic features (16-18). Moreover, these quantitative metabolic MR parameters may also help predict glioma genetic types and monitor tumor responses to treatments $(19,20)$. Compared to PET examinations, MRI tests are more comprehensive, widely available, and safe.

Recently, increasing attention has been directed to the correlation patterns between different MRI modalities. The inter-modality correlations may reflect vital biological processes and the characterization of tumors. For example, the qualitative biomarker T2/FLAIR mismatch, which describes the consistency between $\mathrm{T} 2$ and $\mathrm{T}_{2}$ FLAIR (T2 fluid-attenuated inversion recovery imaging), is a highly specific imaging biomarker for the isocitrate dehydrogenase (IDH)-mutant, 1p/19q non-co-deleted molecular subtype in lower-grade gliomas $(21,22)$. Moreover, previous studies have demonstrated the feasibility of using inter-imagingmodality correlations for assessing tumor prognosis. For example, the higher pixel-wise correlation between the PET signal and the apparent diffusion coefficient (ADC) value from MRIs suggests a shorter time progression $(8,23)$. Hence, exploring inter-metabolite correlations with noninvasive MRI/MRSI contrasts may lead to an improved understanding of gliomas.

Our previous study found that APT contrast and MTC were significantly correlated with the tCho and tNAA (24). However, the group-level correlation analysis might be confounded by inter-subject heterogeneity. Since the inter-metabolite correlations at the individual level have not been systematically investigated, this current study examined the intra-subject inter-metabolite correlation with MRI/MRSI contrasts and utilized intermetabolite correlation coefficients (IMCCs) to differentiate cancer malignancy and proliferation in clinical glioma patients. 


\section{Methods}

\section{Patients}

This retrospective study was approved by the institutional review board, and all activities during this study abided by the basic principles of the Helsinki Declaration. Between June 2015 and September 2018, a total of 145 scans were performed using a 3.0 Tesla MR750 scanner (GE Medical Systems, Milwaukee, WI, USA) with a 32-channel coil. Informed consent was obtained from each participant before scanning. During the scan, patients were instructed to hold their heads still, while a soft sponge mat was used to comfort and reduce potential head movement. The inclusion criteria were as follows: (I) patients scanned with anatomical MRI, CEST, and MRSI; and (II) primary gliomas confirmed by pathological examinations. The following exclusion criteria were applied: (I) unsatisfactory MRSI or CEST scans (14 cases), including mismatched imaging slices between CEST and MRS, severe head motion, and no CEST or MRSI scans; (II) lesions with an insufficient number of voxels (less than 9 voxels) in the MRSI (18 cases); (III) non-tumors confirmed by histopathological examination (6 cases); (IV) patients not receiving surgical operations (8 cases) and duplicated scans (1 case); (V) patients with no gliomas, including 5 cases of lymphomas (1 non-Hodgkin's lymphoma and 4 diffuse large B cell lymphoma), 7 cases of meningiomas, and 1 case of metastasis tumor; and (VI) no primary gliomas, including 17 cases of recurrent gliomas, 3 cases of treatment injuries confirmed at 6-month follow-up, and 1 recurrent primitive neuroectodermal tumor. Following the selection criteria, a total of 17 negative controls (NCs) and 64 glioma patients were enrolled in this study, including 39 LGGs (2 grade I and 37 grade II gliomas) and 25 HGGs (13 grade III and 12 grade IV gliomas).

\section{Scanning protocols}

Anatomical MRI, CEST, and MRSI were conducted. Anatomical MRI scans included T2 fluid-attenuated inversion recovery imaging ( $\mathrm{T}_{2}$ FLAIR), T1 fluidattenuated inversion recovery imaging ( $\mathrm{T}_{1}$ FLAIR), T2weighted fast spin-echo ( $\left.\mathrm{T}_{2} \mathrm{FSE}\right)$, and $\mathrm{T}_{1}$ post-contrast (T1C). All anatomical MRI scans were collected with the same field of view (FOV, $240 \times 240 \mathrm{~mm}^{2}$ ), slice thickness $(5.0 \mathrm{~mm})$, slice spacing $(1.5 \mathrm{~mm})$, and a total of 20 slices covering the whole brain. Both CEST and MRSI were scanned on the same slices: the largest transverse slice of the solid tumor parts and the centrum semiovale in healthy volunteers. For CEST, image acquisition with 2 numbers of excitations was implemented before T1C scanning. A frequency list of $+15.6, \pm 6, \pm 5, \pm 4.5, \pm 4, \pm 3.75, \pm 3.5$, $\pm 3.25, \pm 3, \pm 2.5, \pm 2, \pm 1.5, \pm 1, \pm 0.75, \pm 0.5, \pm 0.25$, and 0 ppm was acquired to collect 64 images and 2 reference images without saturation. The radiofrequency $(\mathrm{RF})$ saturation pulse train consisted of 4 pulses at $2 \mu \mathrm{T}$ amplitude and $400 \mathrm{~ms}$ duration for each. Other CEST imaging parameters are listed as followed: repetition time/echo time $(\mathrm{TR} / \mathrm{TE})=3,000 / 22.6 \mathrm{~ms}$, matrix size $128 \times 128$, slice thickness $5 \mathrm{~mm}$, and FOV $240 \times 240 \mathrm{~mm}^{2}$. The total acquisition time for the CEST MRI of a single transverse brain slice was 3 minutes 18 seconds. For MRSI, twodimensional multi-voxel MRS was implemented with point-resolved spectroscopy (PRESS) pulse sequence with the following parameters: $\mathrm{TR}=1,000 \mathrm{~ms}, \mathrm{TE}=144 \mathrm{~ms}$, FOV $240 \times 240 \mathrm{~mm}^{2}$ with the matrix size of $128 \times 128$, slice thickness $14 \mathrm{~mm}$, and NEX $=0.8$. The voxel size was $7.5 \times 7.5 \times 14 \mathrm{~mm}^{3}$, with a total scanning time of 4 minutes 20 seconds. Areas of hemorrhage, calcification, cystic components, and large vessels were carefully avoided to reduce the potential interference with MRSI, and saturation slabs were applied in 6 directions to reduce potential fold-over and motion artifacts.

\section{Data processing}

The scanned data were transferred to an AW4.6 workstation (AW462) with post-processing capability. For the MRSIs, the following steps were included in the preprocessing: filtering to reduce noise, zero-filling the signal, water signal suppression, signal translating from the time domain to frequency domain with fast Fourier transformation, baseline correction, and phase correction. The total choline/ total creatine $(\mathrm{tCho} / \mathrm{tCr})$ and total $\mathrm{N}$-acetylaspartate/ total creatine (tNAA/tCr) for each voxel were quantified as ratios of the areas of tCho, tNAA, and $\mathrm{tCr}$ peaks which were calculated via curve fitting using the post-processing program from the scanner workstation. Amide proton transfer (APT), with the asymmetric magnetization transfer ratio at $3.5 \mathrm{ppm}$, was derived from the raw CEST data, $\mathrm{B}_{0^{-}}$ shifted by fitting the $\mathrm{Z}$-spectrum according to the report by Zhou et al. (25). The quantitative parameter of the MTC, that is, the magnetization transfer ratio, was calculated using the following equation $\left(S_{0}-S_{\text {saturation }}\right) / S_{0}$, where $S_{\text {saturation }}$ is the signal intensity at $+15.6 \mathrm{ppm}$, and $\mathrm{S}_{0}$ is the signal with no saturation (26). 
A The Included MR sequences and multi-metabolites parameters in within-subject analyses

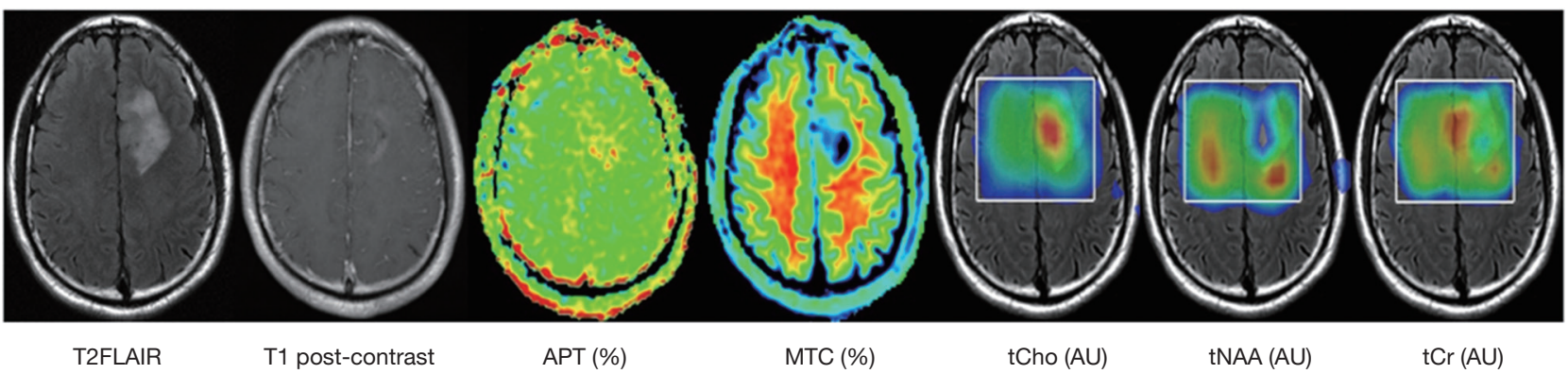

B

The coregistration between MRSI and MRI contrast map

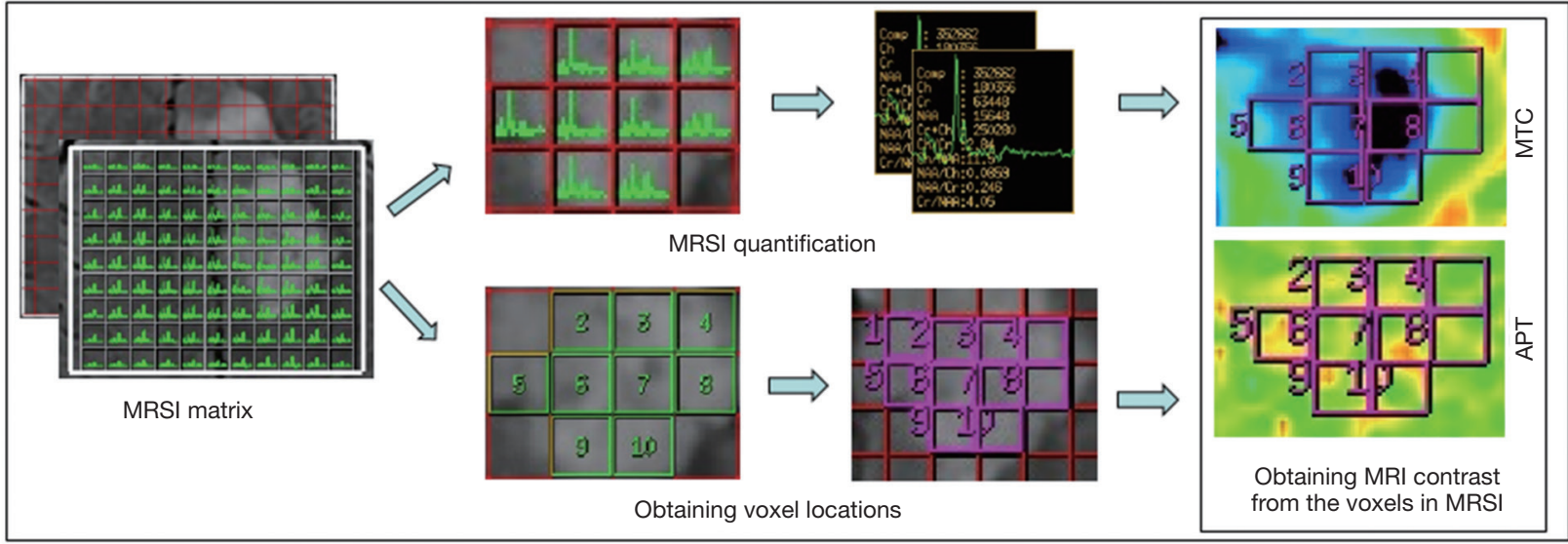

Figure 1 Analysis of the MRSI and MRI parameters and the co-registration flow chart. (A) Representative multi-parametric maps from MRSI and CEST sequences in gliomas. Signals from MRSI (tCho, tNAA, tCr) are color-coded and overlayed on T2-FLAIR images. (B) The flowchart illustrates the co-registration between the MRSI and MRI contrast maps. The MRSI was quantified according to the acquisition matrix and the voxel locations in reference to the MRI were obtained. The voxel locations were then used to obtain the corresponding MRI contrast information. MRSI, magnetic resonance spectroscopic imaging; MRI, magnetic resonance imaging; CEST, chemical exchange saturation transfer; AU, arbitrary unit; APT, amide proton transfer; MTC, magnetization transfer contrast; tCho, total choline; $\mathrm{tCr}$, total creatine; tNAA, total N-acetylaspartate.

\section{Multi-voxel segmentation and quantification of metabolites}

Using the AW4.6 workstation, anatomical MRIs were automatically matched with MRSI scanning slabs, and quantitative parameters were reconstructed according to the acquisition matrix. MRSI voxels that fell completely within the tumor solid parts were included, and voxel-wise quantitative parameters were obtained. After that, voxel positions were recorded concerning anatomical images, based on which corresponding APT and MTC in each voxel were produced. The detailed flowchart of voxel segmentation and co-registration is illustrated in Figure 1.

\section{Histological examinations}

Histological tumor grades were assigned according to
2016 WHO classification criteria on surgically resected specimens (27). Also, immune-histochemical staining was performed on 51 tissue specimens from 64 patients. The Ki-67 labeling index (Ki-67 LI) was used to quantify the tumor cell proliferation status. Ki-67 LI was measured as the percentage of positive cells among all cells counted in the stained area with the highest density of cells. Cells with nuclear staining of any intensity were considered positive.

\section{Statistical analysis}

All data are expressed as mean \pm standard deviation. To explore the inter-metabolite correlation among NCs, LGGs, and HGGs, three inter-metabolites correlation

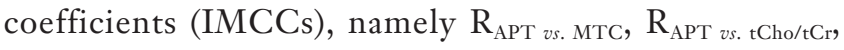


Table 1 The demographic data of the included subjects

\begin{tabular}{lcccc}
\hline Variables & NC & LGGs & HGGs & P value \\
\hline Numbers & 17 & 39 & 25 & N/A \\
Age & $42.47 \pm 11.23$ & $43.38 \pm 13.57$ & $47.2 \pm 12.18$ & 0.40 \\
Gender & & & & 14 \\
Male & 8 & 20 & 11 & 0.85 \\
Female & 9 & 19 & 11 & \\
\hline
\end{tabular}

All data are expressed as mean \pm standard deviation or N. * represents statistical analysis by the Kruskal-Wallis test. No significant differences were observed in patient age and gender composition. NC, healthy control; LGG, low-grade glioma; HGG, high-grade glioma; $\mathrm{N} / \mathrm{A}$, not applicable.

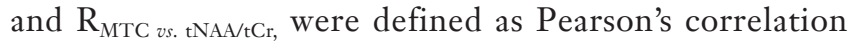
coefficients of APT $v s$. MTC, APT vs. tCho/tCr, and MTC $v s$. $\mathrm{tNAA} / \mathrm{tCr}$, respectively, of solid-tumor voxels in each subject. For example, $\mathrm{R}_{\mathrm{APT} \text { vs. MTC }}$ for a certain patient is the correlation coefficient between APT and MTC contrasts of all tumor voxels from that patient. To investigate the dependency of the IMCCs, regression analysis was conducted between included voxel numbers and the IMCCs. The correlation among the IMCCs and tumor grades was explored with Spearman's rank correlation analysis, and the correlation between each IMCC and Ki67 LI was quantified using Pearson's correlation analysis. To examine the differences of the IMCCs among NC, LGGs, and HGGs, one-way analysis of variance (ANOVA) was implemented, followed by the post hoc analysis of the least-significant difference method. To combine multiIMCCs and remove collinearity IMCCs, multi-variate linear regression or binary logistic regression was used to build combined models to predict the tumor proliferation and grades. The diagnostic performance of the IMCCs was evaluated by the receiver operating characteristic curve (ROC). The differences in the Ki-67 LI between LGGs and HGGs were tested with the independent Student's $t$-test, and the differences in gender composition between NCs, LGGs, and HGGs were tested using the Kruskal-Wallis test. All statistical analyses were performed with either SPSS, IBM 18, or Prism 5.0 GraphPad software, with twotailed $\mathrm{P}$ values $\leq 0.05$ considered statistically significant.

\section{Results}

No significant differences in age or gender were observed in NCs, LGGs, and HGGs (Table 1). HGGs were marked with more rapid cellular proliferative activities. The Ki67 LI in HGGs was significantly higher than that in LGGs
$(26.19 \pm 19.29$ vs. $5.62 \pm 4.56, \mathrm{P}<0.001)$. The total included voxels in NCs, HGGs, LGGs at the group level were 458, 764 , and 558, respectively. The included voxel number of NCs was significantly more than both LGGs and HGGs $(34.65 \pm 2.12$ vs. $19.59 \pm 8.3$ and $22.32 \pm 13.39$, respectively; $\mathrm{P}<0.0001)$. However, no significant correlations were observed between included voxel numbers and $\mathrm{R}_{\mathrm{APT} \text { vs. MTC }}$ $(\mathrm{R}=0.11, \mathrm{P}=0.387), \mathrm{R}_{\mathrm{APT}}$ vs. tho/tCr $(\mathrm{R}=-0.008, \mathrm{P}=0.951)$, and $\mathrm{R}_{\mathrm{MTC} \text { vs } \mathrm{tNAA} / \mathrm{tCr}}(\mathrm{R}=-0.172, \mathrm{P}=0.174)$, indicating that IMCCs might be independent biomarkers.

Compared with NCs, gliomas showed higher absolute IMCC values. However, HGGs demonstrated much greater inter-metabolite correlation. The representative cases of NCs, LGGs, and HGGs are displayed in Figure 2, and the corresponding metabolite correlation scatters are shown in Figure $3 A, B, C . \mathrm{R}_{\mathrm{APT} v \text { s. MTC }}$ and $\mathrm{R}_{\mathrm{APT} v \text { s. } \mathrm{tCho} / \mathrm{Cr}}$ increased with tumor grade. $\mathrm{R}_{\mathrm{APT} \text { vs. MTC }}$ was significantly different in the three groups (NCs vs. LGGs vs. HGGs: $-0.18 \pm 0.38$ vs. $-0.40 \pm 0.34$ vs. $-0.70 \pm 0.29, \mathrm{P}<0.0001$, Figure $3 D)$. $\mathrm{R}_{\mathrm{APT}}$ vs. tCho/tCr $\mathrm{was}$ significantly higher in HGGs compared to NCs $(0.52 \pm 0.32$ vs. $0.01 \pm 0.25, \mathrm{P}<0.0001)$ and LGGs $(0.52 \pm 0.32$ vs. $0.13 \pm 0.34$, $\mathrm{P}<0.0001$ ) (Figure 3D). There appeared to be no significant differences between NCs and LGGs $(\mathrm{P}=0.231$, Figure 3D). Furthermore, no differences in $\mathrm{R}_{\mathrm{MTC} \text { vs. tNAA } \mathrm{tCr}}$ were observed among the three groups $(\mathrm{P}=0.116$, Figure $3 D)$.

$\mathrm{R}_{\mathrm{APT} v \text { s. MTC }}$ and $\mathrm{R}_{\mathrm{APT} \text { vs. tCho/tCr }}$ were significantly correlated with tumor grades with corresponding $\mathrm{R}$ values of -0.41 $(\mathrm{P}=0.001)$ and $0.448(\mathrm{P}<0.001)$, while no significant correlation was observed in $\mathrm{R}_{\mathrm{MTC} \text { vs. } \mathrm{tNAA} / \mathrm{tCr}}(\mathrm{R}=-0.17, \mathrm{P}=0.2)$. With tumor proliferation, only $\mathrm{R}_{\mathrm{APT} \text { vs. MTC }}$ was negatively correlated with $\mathrm{Ki}-67 \mathrm{LI}(\mathrm{R}=-0.33, \mathrm{P}=0.02)$, and neither $\mathrm{R}_{\mathrm{APT} \text { vs. tCho/tCr }}$ nor $\mathrm{R}_{M T C \text { vs. tNAA/tCr }}$ exhibited any significant correlation (Figure 4A,B,C). Predictive models combining different IMCCs were built with regression analysis to evaluate the Ki-67 LI. The combined model, expressed as $\mathrm{Y}$ 

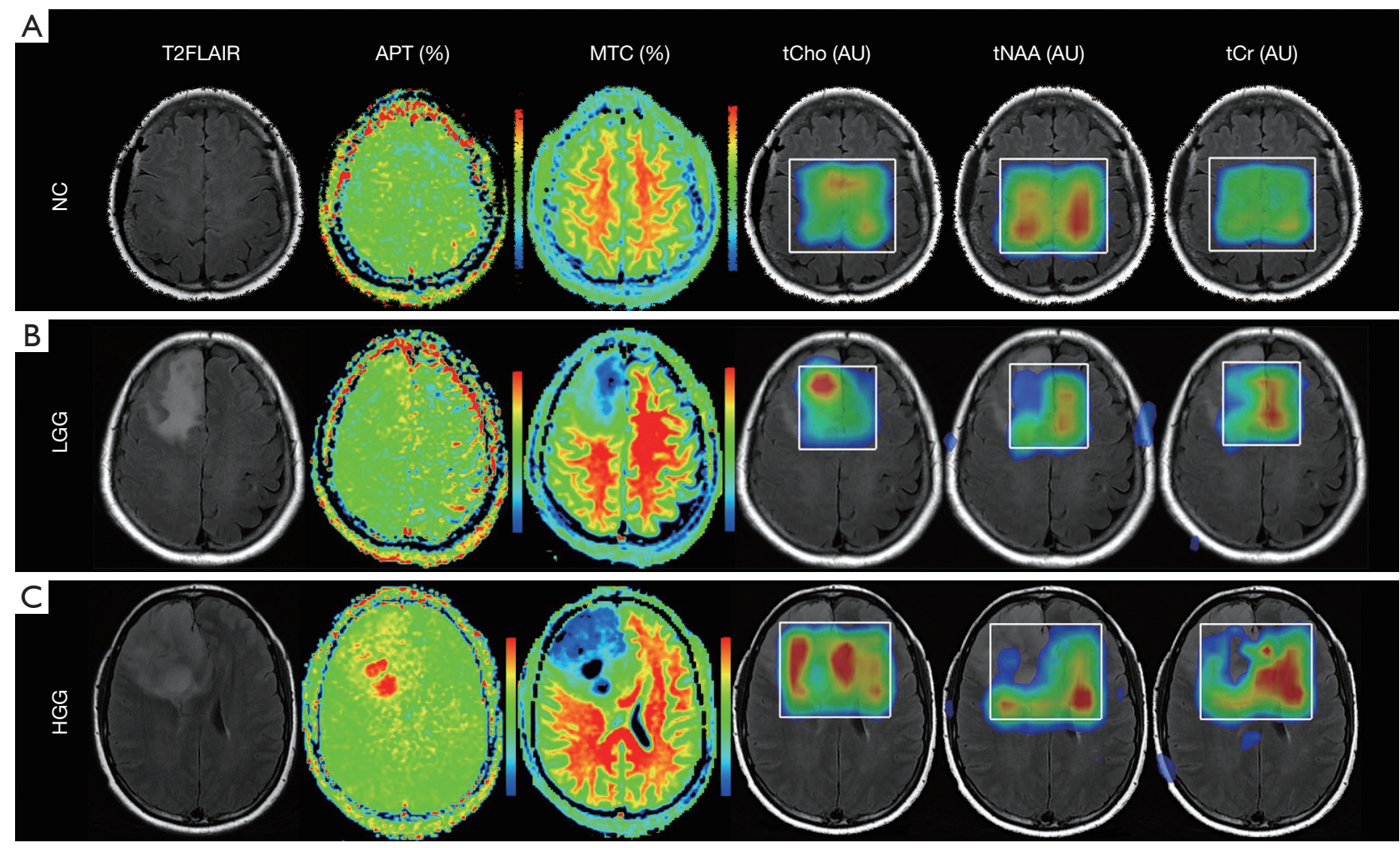

Figure 2 Representative cases of healthy control, low-grade glioma, and high-grade glioma. Representative images from (A) healthy control; (B) grade II glioma patient; and (C) grade IV glioma patient. Compared with healthy controls, gliomas showed increased APT signal and tCho, and reduced MTC signal and tNAA levels. AU, arbitrary unit; NC, healthy control; LGG, low-grade glioma; HGG, highgrade glioma; APT, amide proton transfer; MTC, magnetization transfer contrast; tCho, total choline; tCr, total creatine; tNAA, total $\mathrm{N}$-acetylaspartate.

(predictive Ki-67 expression level) $=12.48-17.08^{*} \mathrm{R}_{\mathrm{APT} \text { vs. MTC }}$ $-14.66^{*} \mathrm{R}_{\mathrm{MTC} v \text { s. tNAA/tCr}}$ displayed improved correlation with Ki-67 LI (R=0.46, P=0.0008, Figure 4D).

In distinguishing between healthy and tumor status,

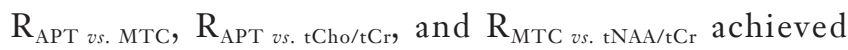
areas under the curve (AUCs) of 0.754, 0.71, and 0.637, respectively (Figure 5A). However, relatively low AUCs $\left(\mathrm{R}_{\mathrm{APT} v \text { v. MTC }} 0.66 ; \mathrm{R}_{\mathrm{APT} v s . \mathrm{tCho} / \mathrm{tCr}} 0.60 ; \mathrm{R}_{\mathrm{MTC} v \text { s. tNAA/tCr }} 0.69\right)$ were obtained in differentiating NCs from LGGs. The differentiation between NCs and HGGs, $\mathrm{R}_{\mathrm{APT} \text { vs. MTC }}$ and

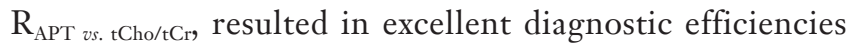
with AUCs of 0.90 and 0.88 , respectively. However, $\mathrm{R}_{\mathrm{MTC} v \mathrm{ss} \mathrm{tNAA} / \mathrm{tCr}}$ showed poor diagnostic performance with an AUC of 0.55. In differentiating LGGs and HGGs, comparable AUCs were obtained in $\mathrm{R}_{\mathrm{APT} v \text { s. MTC }}$ and $\mathrm{R}_{\mathrm{APT} \text { vs. tCho/tCr }}(0.77$ and 0.78$)$; however, relatively low AUC with a value of 0.62 was detected in $\mathrm{R}_{\mathrm{MTC} \text { vs. tNAA/tCr }}$ (Figure 5B). Using regression analysis, predictive models were built by combining different IMCCs to provide complementary information in differentiating glioma malignancy. The logistic regression model was expressed as $\ln [(1-\mathrm{Y}) / \mathrm{Y}]=-2.497^{*} \mathrm{R}_{\mathrm{APT} v \text { s. } \mathrm{MTC}}+2.663^{*} \mathrm{R}_{\mathrm{APT} v \text { t } \mathrm{tCho} / \mathrm{tCr}}$ $-2.591^{*} \mathrm{R}_{\mathrm{MTC} \text { vs. } \mathrm{tNAA} / \mathrm{tCr}}-1.595$, in which $\mathrm{Y}$ indicates tumor grade. The AUC of the combined multi-IMCCs model improved to 0.854 , with sensitivity and specificity of $80.0 \%$ and $79.5 \%$, respectively (Figure $5 B$ ).

\section{Discussion}

Compared with normal controls, gliomas showed a stronger inter-metabolites correlation. Quantitative biomarkers of inter-metabolites correlation, that is, the IMCCs, revealed different metabolic correlation patterns in NCs, LGGs, and HGGs. Moreover, some patient-specific IMCCs significantly correlated with tumor grade and Ki-67 LI. The combined multi-IMCCs model could effectively 
A
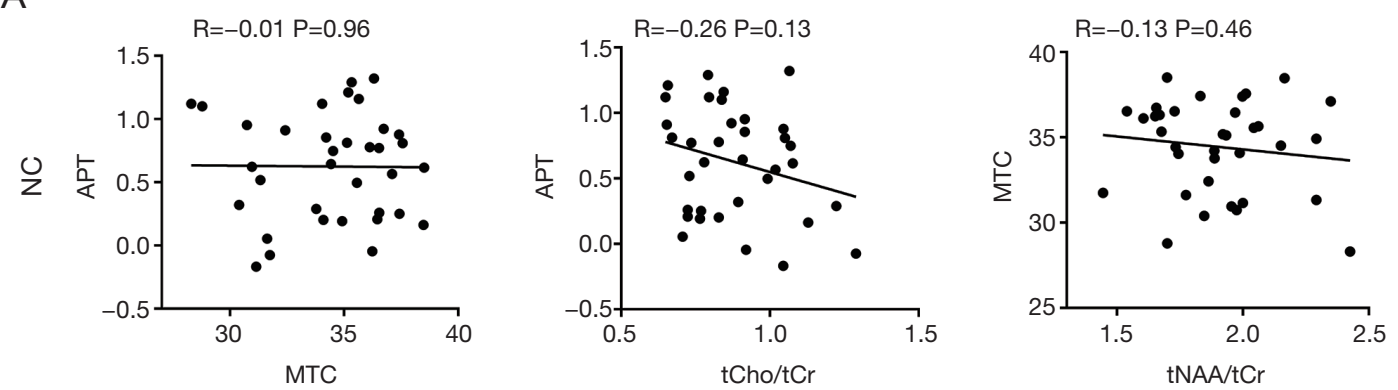

B
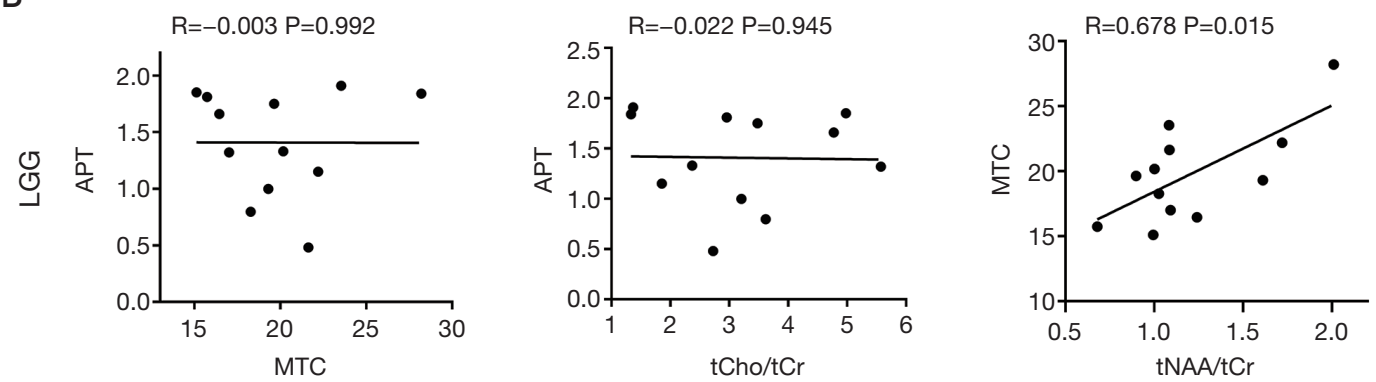

C
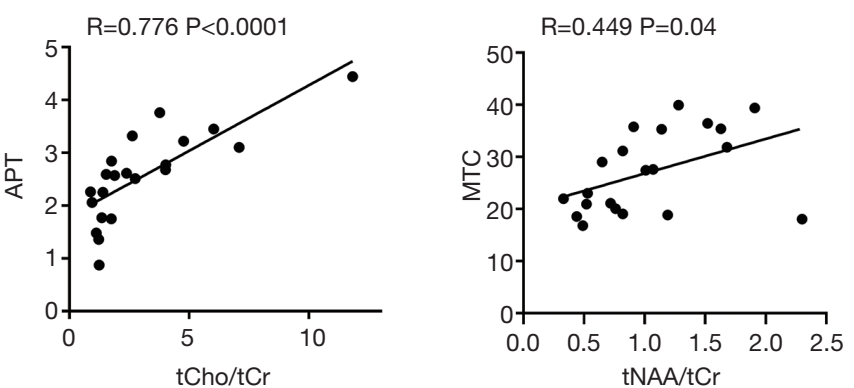

D
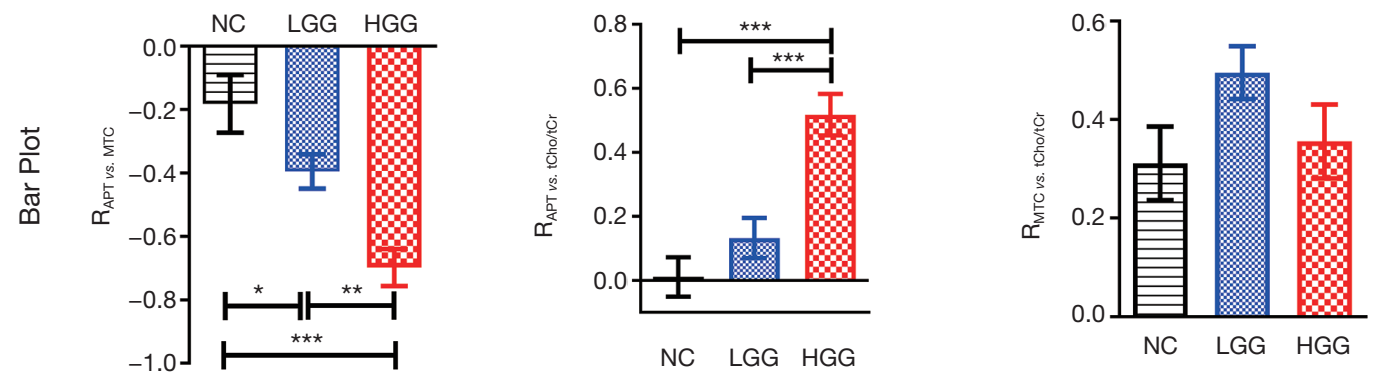

Figure 3 The scatter patterns and bar plots of the inter-metabolite correlation coefficients in healthy controls, low-grade glioma, and highgrade glioma patients. Scatter plots of the IMCCs in (A) healthy control; (B) grade II glioma patient; and (C) grade IV glioma patient. Subjects correspond to those in Figure 2. From left to right, the plots are correlations of APT vs. MTC, APT vs. tCho/tCr, and MTC vs. tNAA/tCr, sequentially. (D) Bar plot of $\mathrm{R}_{\mathrm{APT} \text { vs. MTC }}, \mathrm{R}_{\mathrm{APT} \text { vs. tCho/tCr }}$, and $\mathrm{R}_{\mathrm{MTC} v \text { s. tNAA/tCr }}$ in the three groups. $\mathrm{R}_{\mathrm{APT} \text { vs. MTC }}$ and $\mathrm{R}_{\mathrm{APT} \text { vs. tCho/tCr }}$ are significantly different among the three groups, except in the case of $\mathrm{R}_{\mathrm{APT}} v$ s. $\mathrm{tCho} / \mathrm{tCr}$ in differentiating NCs from LGGs. No significant differences were observed in $\mathrm{R}_{\mathrm{MTC} \text { vs. tNAA/tCr }}$ among the three subject groups. IMCC, inter-metabolite correlation coefficient; NC, healthy control; LGG, low-grade glioma; HGG, high-grade glioma; APT, amide proton transfer; MTC, magnetization transfer contrast; tCho, total choline; tCr, total creatine; tNAA, total $\mathrm{N}$-acetylaspartate. *, $0.01<\mathrm{P} \leq 0.05 ;{ }^{* *}, 0.001<\mathrm{P} \leq 0.01$; ${ }^{* * *}, \mathrm{P} \leq 0.001$. 
A
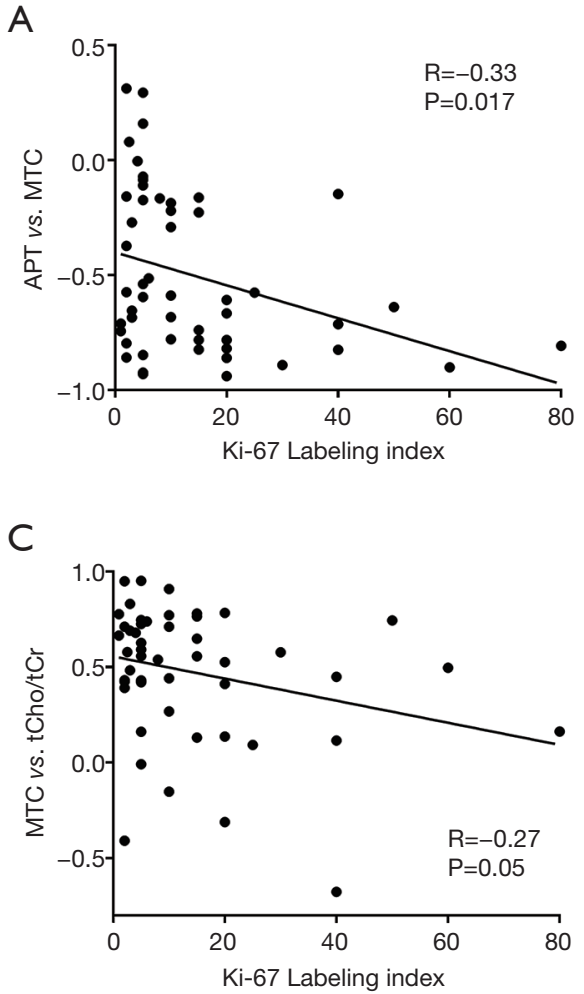

B

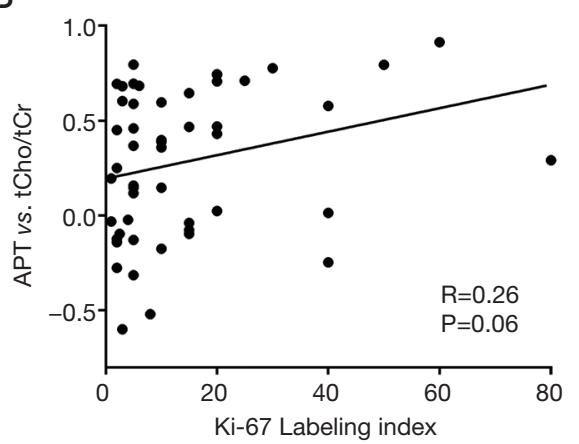

D

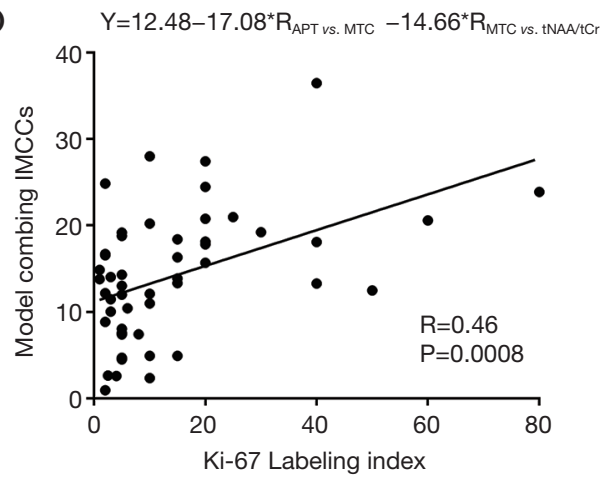

Figure 4 Scatter plots showing the correlations between the inter-metabolite correlation coefficients and the Ki-67 labeling index. Scatter

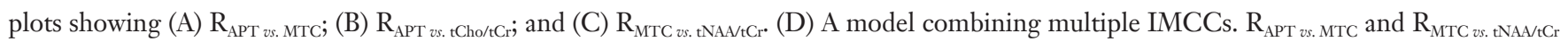
significantly correlated with Ki-67 LI (A and C). The model combining $\mathrm{R}_{\mathrm{APT} \text { vs. MTC }}$ and $\mathrm{R}_{\mathrm{MTC} \text { vs } \mathrm{ENAA} / \mathrm{Cr}}$ showed an improved correlation with the Ki-67 LI with $\mathrm{P}<0.001$ (D). IMCC, inter-metabolite correlation coefficient; APT, amide proton transfer; MTC, magnetization transfer contrast; tCho, total choline; $\mathrm{tCr}$, total creatine; tNAA, total N-acetylaspartate; LI, labeling index.

predict tumor proliferation and differentiate LGGs from HGGs. This may represent a vital non-invasive metabolic biomarker for pre-surgical evaluation of gliomas.

Personalization of metabolic therapies may be an effective cancer treatment strategy. However, this requires the fine-tune matching of therapy to an individual's unique physiology (28). Hence, cancer risk stratification with individual IMCCs might guide personal treatment regimens. As quantified by advanced MRI technologies, tCho, tNAA, APT, and MTC represent total choline concentrations, total $\mathrm{N}$-acetylaspartate, cellular mobile proteins and peptides, and immobile macromolecular substances $(3,5,15,29,30)$. The ratios of $\mathrm{tCho} / \mathrm{tCr}$ and $\mathrm{tNAA} /$ $\mathrm{tCr}$ are frequently used to evaluate tumor burden and losses of neurons, respectively (31). The rapid growth of tumors increases the demands on metabolic substances, which leads to the accumulation of choline and an increase in mobile peptide and protein concentrations or the APT signal $(25,32)$. The invasiveness of tumors on brain tissues causes the reduction of tNAA and immobile macromolecular substances $(13,32)$. Thus, to identify the different metabolic statuses in patients with gliomas, it is meaningful to correlate APT with MTC, $\mathrm{APT}$ with $\mathrm{tCho} / \mathrm{tCr}$, and MTC with $\mathrm{tNAA} / \mathrm{tCr}$.

Moreover, the rapid growth of the tumor relies on a higher metabolic turnover, and it has been shown that the metabolism of many substances, such as peptides, lipids, and carbohydrates, accelerates during carcinogenesis $(30,33)$. Due to different cell proliferation rates, metabolite transformation rates in tumor tissue differ according to the tumor malignancy, contributing to a different IMCC for each patient. Hence, patient-specific IMCC allows the individual evaluation of metabolite correlation in different tumor types.

The complex metabolic correlations in tumor tissue could be a vital pathophysiological basis for seeking useful biomarkers that reflect tumor metabolic procedures (34). The current study identified multi-quantitative metabolite- 

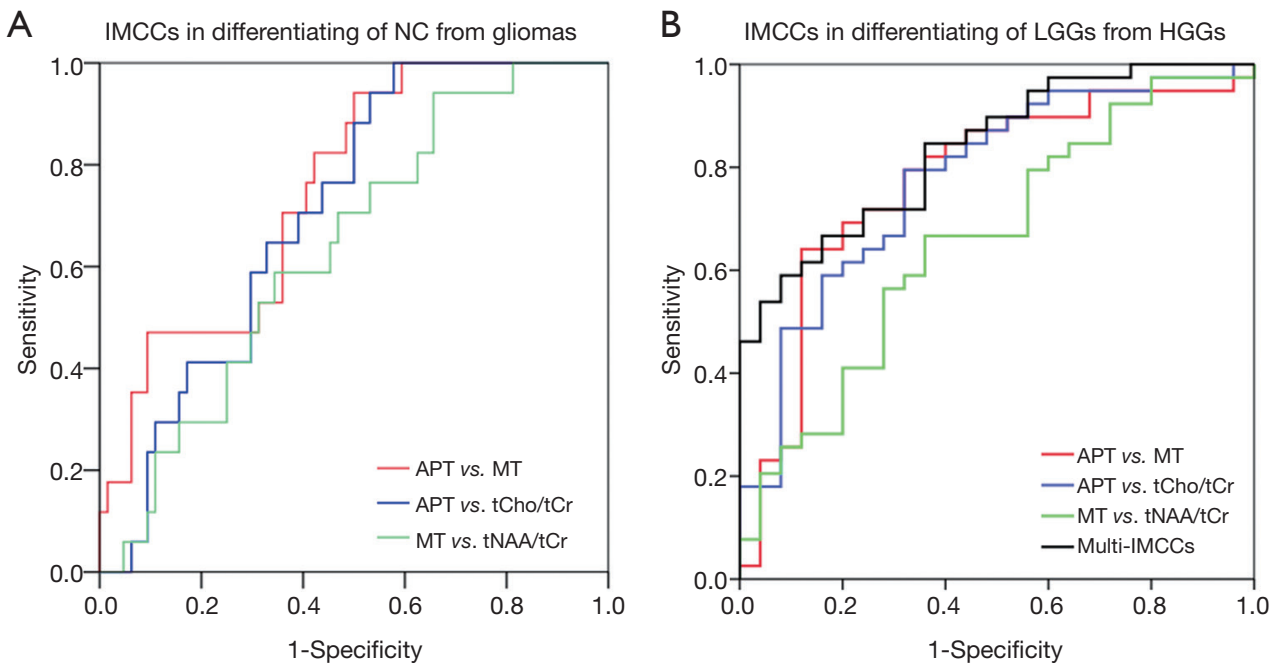

Figure 5 Diagnostic performances of the inter-metabolite correlation coefficients in differentiating healthy controls from gliomas, and lowgrade gliomas from high-grade gliomas. (A) ROC curves of the IMCCs in differentiating NCs and gliomas. $\mathrm{R}_{\mathrm{APT} v \text { s. MTC }}$ showed the best AUC value of 0.754. (B) ROC curves for the differentiation of LGGs from HGGs. $\mathrm{R}_{\mathrm{APT} \text { vs. } \mathrm{tCho} / \mathrm{t} \mathrm{Cr}}$ showed the best AUC of 0.78 . Combining multiple IMCCs resulted in improved diagnostic power with an AUC of 0.854. IMCC, inter-metabolite correlation coefficient; NC, healthy control; LGG, low-grade glioma; HGG, high-grade glioma; APT, amide proton transfer; MTC, magnetization transfer contrast; tCho, total choline; tCr, total creatine; tNAA, total N-acetylaspartate; ROC, receiver operating characteristic; AUC, area under the curve.

related biomarkers for glioma evaluation. Moreover, different IMCCs can be used to evaluate different aspects in stratifying tumors. For example, $\mathrm{R}_{\mathrm{APT} \text { vs. } \mathrm{tCh} / \mathrm{tCr}}$ reflects the correlation of choline (an indicator of tumor cell membrane formation) and mobile peptides and proteins, and $\mathrm{R}_{M T C \text { vs. } \mathrm{tNAA} / \mathrm{CCr}}$ reflects the semisolid macromolecular substance (indicating the integrity of the brain structure) and tNAA decrease (a biomarker of neuron loss) (25,31). Different tumor malignancies contribute to varied pathological processes, and noninvasive IMCCs allow the quantitative description of those processes. Thus, the combination of multi-IMCCs provides a more effective index for predicting tumor proliferation and differentiating tumor grades. Also, intra-subject IMCCs can be more specific in differentiation LGGs and HGGs compared to group-level correlation analysis (35).

IMCCs, such as $\mathrm{R}_{\mathrm{APT} \text { vs. MTC, }}$ are novel biomarkers for glioma malignancy and proliferation. Previously, we reported a positive correlation between APT contrast, tumor grades, and Ki-67 LI (17). While the present study demonstrated a negative correlation between $\mathrm{R}_{\mathrm{APT} \text { vs. MTC }}$ and tumor grade or Ki-67 LI, this does not conflict with the previous study since $\mathrm{R}_{\mathrm{APT} \text { vs. MTC }}$ represents the correlation coefficient between the inter-metabolites APT and MTC. $\mathrm{R}_{\mathrm{APT} \text { vs. MTC }}$ is a different signal compared to APT contrast itself. IMCCs may be less influenced by inter-subject and spatial tumor heterogeneity compared to studies using metabolic imaging contrasts themselves for differentiating glioma malignancy (35). Therefore, IMCCs may be effective novel biomarkers for differentiating glioma malignancy and proliferation.

Three IMCCs, namely, $\mathrm{R}_{\mathrm{APT} \text { vs. tCho/tCr }}, \mathrm{R}_{\mathrm{APT} \text { vs. MTC, }}$ and $\mathrm{R}_{\mathrm{MTC} \text { vs. tNAA/TCr}}$, were included based on their pathophysiological meanings in this study. APT and tCho/ $\mathrm{tCr}$ were significantly correlated with tumor malignancy and proliferation, while MTC and $\mathrm{tNAA} / \mathrm{tCr}$ represent brain structures and neurons (36). In this study, tCho/tCr and $\mathrm{tNAA} / \mathrm{tCr}$ were examined rather than tCho and $\mathrm{tNAA}$ because $\mathrm{tCho} / \mathrm{tCr}$ and $\mathrm{tNAA} / \mathrm{tCr}$ are considered more reliable than tCho and tNAA alone. Moreover, a relatively short time of repetition ( $\mathrm{TR}=1,000 \mathrm{~ms}$ ) and a relatively long echo time (TE $=144 \mathrm{~ms}$ ) were utilized in our MRSI protocols. A relatively short TR can reduce the scanning time, allowing more averages within a clinically feasible scanning period. Meanwhile, a longer TE can preferably suppress the signals from macromolecules to achieve more stable spectroscopic baselines, contributing to more reliable quantifications $(37,38)$. As demonstrated in this study results, the IMCCs showed no significant correlation with the number of included voxels. The inter-metabolite correlation analyses may have been less sensitive to tumor 
size and spatial location.

There are several limitations to this study. First, while the study demonstrated the adequacy of single-slice MRI and MRSI for glioma grading, three-dimensional APT and MRSI might provide more comprehensive information regarding cancers' spatial heterogeneity. Second, the mismatch in slice thickness between MRSI and CEST MRI may have caused some inaccuracy in our quantification. Third, $I D H$ gene status and the $1 \mathrm{p} 19 \mathrm{q}$ codeletion were not measured in the dataset included in this study. Considering the increasingly important roles of these latter genetic biomarkers in glioma risk-stratification and treatment guidance $(39,40)$, future correlation analyses among IMCCs, IDH status, and $1 \mathrm{p} / 19 \mathrm{q}$ codeletion may provide further diagnostic information in the clinical setting.

\section{Conclusions}

Compared with normal controls, IMCCs generally showed an increase in glioma malignancy, and some IMCCs were effective at predicting tumor proliferation, and combining multiple IMCCs further improved cancer characterization. In conclusion, IMCCs might be an important novel clinical biomarker for the non-invasive characterization of gliomas in patients.

\section{Acknowledgments}

The authors thank Dr. Yang Fan from GE Healthcare for his general advice on preparation of the manuscript.

Funding: This work was supported by grants from the National Program of the Ministry of Science and Technology of China during the "12th Five-Year Plan" (ID: 2011BAI08B10) and the National Natural Science Foundation of China (No. 81171308, No. 81570462, and No. 81730049).

\section{Footnote}

Conflicts of Interest: All authors have completed the ICMJE uniform disclosure form (available at http://dx.doi. org/10.21037/qims-20-1163). The authors have no conflicts of interest to declare.

Ethical Statement: This retrospective study was approved by the institutional review board, and all activities during this study abided by the basic principles of the Helsinki Declaration. Informed consent was obtained from each participant before scanning.

Open Access Statement: This is an Open Access article distributed in accordance with the Creative Commons Attribution-NonCommercial-NoDerivs 4.0 International License (CC BY-NC-ND 4.0), which permits the noncommercial replication and distribution of the article with the strict proviso that no changes or edits are made and the original work is properly cited (including links to both the formal publication through the relevant DOI and the license). See: https://creativecommons.org/licenses/by-nc-nd/4.0/.

\section{References}

1. Wen PY, Kesari S. Malignant gliomas in adults. N Engl J Med 2008;359:492-507.

2. Stupp R, Mason WP, van den Bent MJ, Weller M, Fisher B, Taphoorn MJ, Belanger K, Brandes AA, Marosi C, Bogdahn U, Curschmann J, Janzer RC, Ludwin SK, Gorlia T, Allgeier A, Lacombe D, Cairncross JG, Eisenhauer E, Mirimanoff RO; European Organisation for Research and Treatment of Cancer Brain Tumor and Radiotherapy Groups; National Cancer Institute of Canada Clinical Trials Group. Radiotherapy plus concomitant and adjuvant temozolomide for glioblastoma. N Engl J Med 2005;352:987-96.

3. Ramli N, Khairy AM, Seow P, Tan LK, Wong JH, Ganesan D, Rahmat K. Novel application of chemical shift gradient echo in- and opposed-phase sequences in $3 \mathrm{~T}$ MRI for the detection of H-MRS visible lipids and grading of glioma. Eur Radiol 2016;26:2019-29.

4. Karasinska JM, Topham JT, Kalloger SE, Jang GH, Denroche RE, Culibrk L, Williamson LM, Wong HL, Lee MKC, O'Kane GM, Moore RA, Mungall AJ, Moore MJ, Warren C, Metcalfe A, Notta F, Knox JJ, Gallinger S, Laskin J, Marra MA, Jones SJM, Renouf DJ, Schaeffer DF. Altered Gene Expression along the Glycolysis-Cholesterol Synthesis Axis Is Associated with Outcome in Pancreatic Cancer. Clin Cancer Res 2020;26:135-46.

5. Dhermain FG, Hau P, Lanfermann H, Jacobs AH, van den Bent MJ. Advanced MRI and PET imaging for assessment of treatment response in patients with gliomas. Lancet Neurol 2010;9:906-20.

6. Hua T, Zhou W, Zhou Z, Guan Y, Li M. Heterogeneous parameters based on (18)F-FET PET imaging can non-invasively predict tumor grade and isocitrate dehydrogenase gene 1 mutation in untreated gliomas. Quant Imaging Med Surg 2021;11:317-27. 
7. Cicone F, Carideo L, Scaringi C, Arcella A, Giangaspero F, Scopinaro F, Minniti G. (18)F-DOPA uptake does not correlate with IDH mutation status and $1 \mathrm{p} / 19 \mathrm{q}$ codeletion in glioma. Ann Nucl Med 2019;33:295-302.

8. Gerstner ER. MRI and PET: Noninvasive Tools to Probe the Biology of Diffuse Intrinsic Pontine Glioma. J Nucl Med 2017;58:1262-3.

9. Kebir S, Lazaridis L, Weber M, Deuschl C, Stoppek AK, Schmidt T, Monninghoff C, Blau T, Keyvani K, Umutlu L, Pierscianek D, Forsting M, Stuschke M, Antoch G, Sure U, Kleinschnitz C, Scheffler B, Colletti PM, Rubello D, Herrmann K, Glas M. Comparison of L-Methyl-11CMethionine PET With Magnetic Resonance Spectroscopy in Detecting Newly Diagnosed Glioma. Clin Nucl Med 2019;44:e375-e381.

10. Emwas AH, Roy R, McKay RT, Tenori L, Saccenti E, Gowda GAN, Raftery D, Alahmari F, Jaremko L, Jaremko M, Wishart DS. NMR Spectroscopy for Metabolomics Research. Metabolites 2019;9:123.

11. Ward KM, Aletras AH, Balaban RS. A new class of contrast agents for MRI based on proton chemical exchange dependent saturation transfer (CEST). J Magn Reson 2000;143:79-87.

12. Dou W, Lin CE, Ding H, Shen Y, Dou C, Qian L, Wen $\mathrm{B}, \mathrm{Wu}$ B. Chemical exchange saturation transfer magnetic resonance imaging and its main and potential applications in pre-clinical and clinical studies. Quant Imaging Med Surg 2019;9:1747-66.

13. Pui MH. Magnetization transfer analysis of brain tumor, infection, and infarction. J Magn Reson Imaging 2000;12:395-9.

14. Zheng Y, Wang X, Zhao X. Magnetization Transfer and Amide Proton Transfer MRI of Neonatal Brain Development. Biomed Res Int 2016;2016:3052723.

15. Zhou J, Heo HY, Knutsson L, Zijl PCMV, Jiang S. APT weighted MRI: Techniques, current neuro applications, and challenging issues. Journal of Magnetic Resonance Imaging 2019;50:347-64.

16. Park JE, Kim HS, Park KJ, Kim SJ, Kim JH, Smith SA. Pre- and Posttreatment Glioma: Comparison of Amide Proton Transfer Imaging with MR Spectroscopy for Biomarkers of Tumor Proliferation. Radiology 2016;278:514-23.

17. Su C, Liu C, Zhao L, Jiang J, Zhang J, Li S, Zhu W, Wang J. Amide Proton Transfer Imaging Allows Detection of Glioma Grades and Tumor Proliferation: Comparison with Ki-67 Expression and Proton MR Spectroscopy Imaging. AJNR Am J Neuroradiol 2017;38:1702-9.
18. Joo B, Han K, Ahn SS, Choi YS, Chang JH, Kang SG, Kim SH, Zhou J, Lee SK. Amide proton transfer imaging might predict survival and IDH mutation status in highgrade glioma. Eur Radiol 2019;29:6643-52.

19. Natsumeda $M$, Igarashi $H$, Nomura $T$, Ogura $R$, Tsukamoto Y, Kobayashi T, Aoki H, Okamoto K, Kakita A, Takahashi H, Nakada T, Fujii Y. Accumulation of 2-hydroxyglutarate in gliomas correlates with survival: a study by 3.0-tesla magnetic resonance spectroscopy. Acta Neuropathol Commun 2014;2:158.

20. Fellah S, Caudal D, De Paula AM, Dory-Lautrec P, Figarella-Branger D, Chinot O, Metellus $\mathrm{P}$, Cozzone PJ, Confort-Gouny S, Ghattas B, Callot V, Girard N. Multimodal MR imaging (diffusion, perfusion, and spectroscopy): is it possible to distinguish oligodendroglial tumor grade and $1 \mathrm{p} / 19 \mathrm{q}$ codeletion in the pretherapeutic diagnosis? AJNR Am J Neuroradiol 2013;34:1326-33.

21. Patel SH, Poisson LM, Brat DJ, Zhou Y, Cooper L, Snuderl M, Thomas C, Franceschi AM, Griffith B, Flanders AE, Golfinos JG, Chi AS, Jain R. T2-FLAIR Mismatch, an Imaging Biomarker for IDH and $1 \mathrm{p} / 19 \mathrm{q}$ Status in Lower-grade Gliomas: A TCGA/TCIA Project. Clin Cancer Res 2017;23:6078-85.

22. Broen MPG, Smits M, Wijnenga MMJ, Dubbink HJ, Anten M, Schijns O, Beckervordersandforth J, Postma AA, van den Bent MJ. The T2-FLAIR mismatch sign as an imaging marker for non-enhancing IDH-mutant, 1p/19qintact lower-grade glioma: a validation study. Neuro Oncol 2018;20:1393-9.

23. Zukotynski KA, Vajapeyam S, Fahey FH, Kocak M, Brown D, Ricci KI, Onar-Thomas A, Fouladi M, Poussaint TY. Correlation of (18)F-FDG PET and MRI Apparent Diffusion Coefficient Histogram Metrics with Survival in Diffuse Intrinsic Pontine Glioma: A Report from the Pediatric Brain Tumor Consortium. J Nucl Med 2017;58:1264-9.

24. Liu C, Zhang S, Yao Y, Su C, Wang Z, Wang M, Zhu W. Associations Between Diffusion Dynamics and Functional Outcome in Acute and Early Subacute Ischemic Stroke. Clin Neuroradiol 2020;30:517-24.

25. Zhou J, Zhu H, Lim M, Blair L, Quinones-Hinojosa A, Messina SA, Eberhart CG, Pomper MG, Laterra J, Barker PB, van Zijl PC, Blakeley JO. Three-dimensional amide proton transfer MR imaging of gliomas: Initial experience and comparison with gadolinium enhancement. J Magn Reson Imaging 2013;38:1119-28.

26. Stanisz GJ, Odrobina EE, Pun J, Escaravage M, Graham SJ, Bronskill MJ, Henkelman RM. T1, T2 relaxation and 
magnetization transfer in tissue at 3T. Magn Reson Med 2005;54:507-12.

27. Louis DN, Perry A, Reifenberger G, von Deimling A, Figarella-Branger D, Cavenee WK, Ohgaki H, Wiestler OD, Kleihues P, Ellison DW. The 2016 World Health Organization Classification of Tumors of the Central Nervous System: a summary. Acta Neuropathologica 2016;131:803-20.

28. Seyfried TN, Flores RE, Poff AM, D'Agostino DP. Cancer as a metabolic disease: implications for novel therapeutics. Carcinogenesis 2014;35:515-27.

29. Sun J, Chen P, Bi C. 1H-MRS technique and spectroscopic imaging LCModel based adolescent obese metabolic syndrome research. Multimedia Tools and Applications 2016;76:19491-505.

30. Spratlin JL, Serkova NJ, Eckhardt SG. Clinical applications of metabolomics in oncology: a review. Clin Cancer Res 2009;15:431-40.

31. Howe FA, Barton SJ, Cudlip SA, Stubbs M, Saunders DE, Murphy M, Wilkins P, Opstad KS, Doyle VL, McLean MA, Bell BA, Griffiths JR. Metabolic profiles of human brain tumors using quantitative in vivo $1 \mathrm{H}$ magnetic resonance spectroscopy. Magn Reson Med 2003;49:223-32.

32. Griffin JL, Shockcor JP. Metabolic profiles of cancer cells. Nat Rev Cancer 2004;4:551-61.

33. Pandey R, Caflisch L, Lodi A, Brenner AJ, Tiziani S. Metabolomic signature of brain cancer. Mol Carcinog 2017;56:2355-71.

34. Sullivan MR, Danai LV, Lewis CA, Chan SH, Gui DY, Kunchok T, Dennstedt EA, Vander Heiden MG, Muir

Cite this article as: Su C, Li S, Chen X, Liu C, Shaghaghi M, Jiang J, Zhang S, Qin Y, Cai K. Predicting cancer malignancy and proliferation in glioma patients: intra-subject intermetabolite correlation analyses using MRI and MRSI contrast scans. Quant Imaging Med Surg 2021;11(6):2721-2732. doi: 10.21037/qims-20-1163
A. Quantification of microenvironmental metabolites in murine cancers reveals determinants of tumor nutrient availability. Elife 2019;8:27.

35. Su C, Zhao L, Li S, Jiang J, Cai K, Shi J, Yao Y, Ao Q, Zhang G, Shen N, Hu S, Zhang J, Qin Y, Zhu W. Amid proton transfer (APT) and magnetization transfer (MT) MRI contrasts provide complimentary assessment of brain tumors similarly to proton magnetic resonance spectroscopy imaging (MRSI). Eur Radiol 2019;29:1203-10.

36. Roell SA, Dreher W, Busch E, Leibfritz D. Magnetization transfer attenuates metabolite signals in tumorous and contralateral animal brain: in vivo observations by proton NMR spectroscopy. Magn Reson Med 1998;39:742-8.

37. Li Y, Lafontaine M, Chang S, Nelson SJ. Comparison between Short and Long Echo Time Magnetic Resonance Spectroscopic Imaging at 3T and 7T for Evaluating Brain Metabolites in Patients with Glioma. ACS Chem Neurosci 2018;9:130-7.

38. Li Y, Srinivasan R, Ratiney H, Lu Y, Chang SM, Nelson SJ. Comparison of T(1) and T(2) metabolite relaxation times in glioma and normal brain at 3T. J Magn Reson Imaging 2008;28:342-50.

39. Yan H, Parsons DW, Jin G, McLendon R, Rasheed BA, Yuan W, Kos I, Batinic-Haberle I, Jones S, Riggins GJ, Friedman H, Friedman A, Reardon D, Herndon J, Kinzler KW, Velculescu VE, Vogelstein B, Bigner DD. IDH1 and IDH2 mutations in gliomas. N Engl J Med 2009;360:765-73.

40. Ludwig K, Kornblum HI. Molecular markers in glioma. J Neurooncol 2017;134:505-12. 